\title{
Bridging data and models
}

Jennifer Marlon

GPWG workshop, Petersham, USA, 27 September - 2 October 2015

The Global Paleofire Working Group (GPWG) met for a final synthesis workshop. The meeting reflected the culmination of five concurrent advances during the past decade, including an increase in wildfire salience globally and thus increased research motivation, the development of the Global Charcoal Database (GCD), the emergence of sophisticated global fire models, and the widespread adoption of open-source tools like the "R" programming language. The convergence of these factors enabled 36 researchers from 11 countries to address questions about fire on many temporal and spatial scales, producing insights that would have been unattainable prior to GPWG's creation.

In anticipation of the workshop, a method for gridding the GCD (Marlon et al. 2016) was developed to facilitate data-model comparisons (Fig. 1). The gridded maps provide new spatial information for specific intervals highlighted in the past millennium. Colored dots on the maps are anomalies that indicate increases or decreases in burning relative to average burning at that same location during the base period (1000-1800 CE). Recent human impacts on fire are particularly evident around the turn of the 19th century in eastern Asia, Australasia, and North America.

The limitation of working with anomalies was a key discussion topic at the workshop, prompting three initiatives aimed at quantitatively calibrating paleofire records. Currently, charcoal particles are measured using very diverse methods (e.g. counts, areas, weights, ratios). Using different quantification methods, coupled with the complexities of charcoal production, transportation, and deposition, limits meaningful comparisons across records from different locations. Standardizing paleofire records to

\section{Charcoal Influx z-Scores}
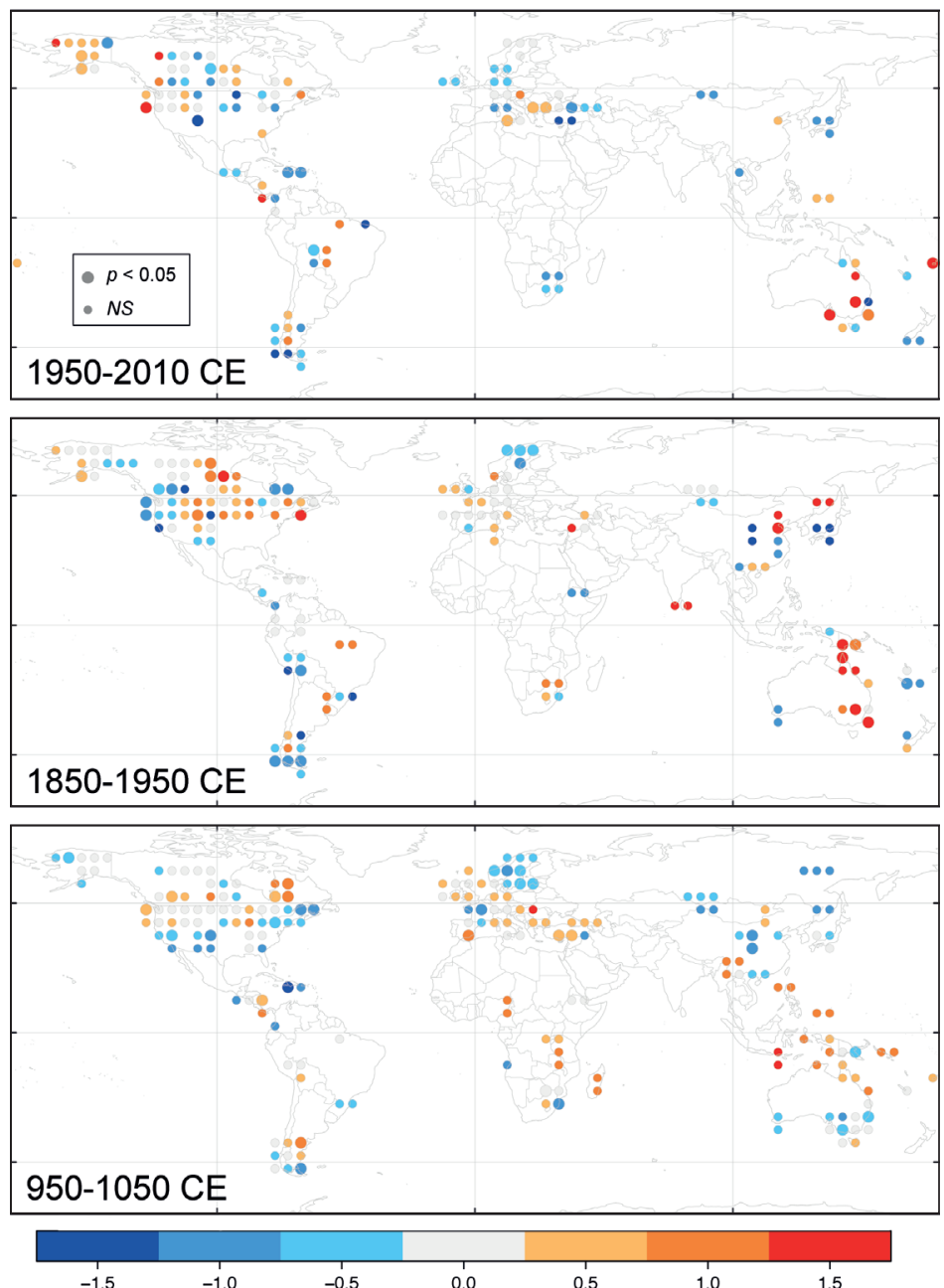

Figure 1: Spatially gridded biomass burning for 1950-2010, 1850-1950, and 950-1050 CE. Charcoal influx anomaly base period is 1000-1800 CE. From Marlon et al. 2016.

a unitless dimension enables comparisons of relative changes through time, but the loss of units prevents analyses of spatial variability. Approaches using a statistical process model, a modern database of charcoal surface samples, and a series of regional multi-proxy comparisons were each developed to enable modeling of, or quantitative comparisons between, charcoal accumulation rates and independent estimates of area burned from fire-scar, historical, and satellite-based data. These efforts will elucidate the factors controlling charcoal accumulation, and may help to initialize and constrain fire simulations at multiple scales.

Workshop projects focused on human-fire interactions, fire ecology, and fire effects on global atmospheric chemistry and climate. Synthesizing anthropological evidence about human fire-use in traditional societies into a quantitative framework is needed to allow for its integration into global fire models. The current generation of models relies on simple assumptions between population density, fire ignitions, and fire suppression that draw from present-day data. Although modeling necessitates some simplification, synthesizing and incorporating data about cultural fire practices will enable model assessment and improvement not only for paleofire simulations, but also for simulations of past land-cover and landuse changes (e.g. through collaboration with PAGES' LandCover6k working group). Such interdisciplinary research is essential for accurately understanding fire, human, and vegetation coupling with the climate system. The ultimate goal is to use this type of systematic and iterative approach of evaluating past fire activity and paleofire simulations to better contextualize contemporary fire management and understand future fire.

GPWG is continuing its work as GPWG2, with a growing emphasis on using data and models together to address research challenges in ecosystem and climate dynamics, and fire management, among others. Future meetings will be announced through the PAGES' website.

\section{AFFILIATIONS}

School of Forestry and Environmental Studies, Yale University, New Haven, USA

\section{CONTACT}

Jennifer Marlon: jennifer.marlon@yale.edu REFERENCES

Marlon JR et al. (2016) Biogeosci 13: 3225-3244 